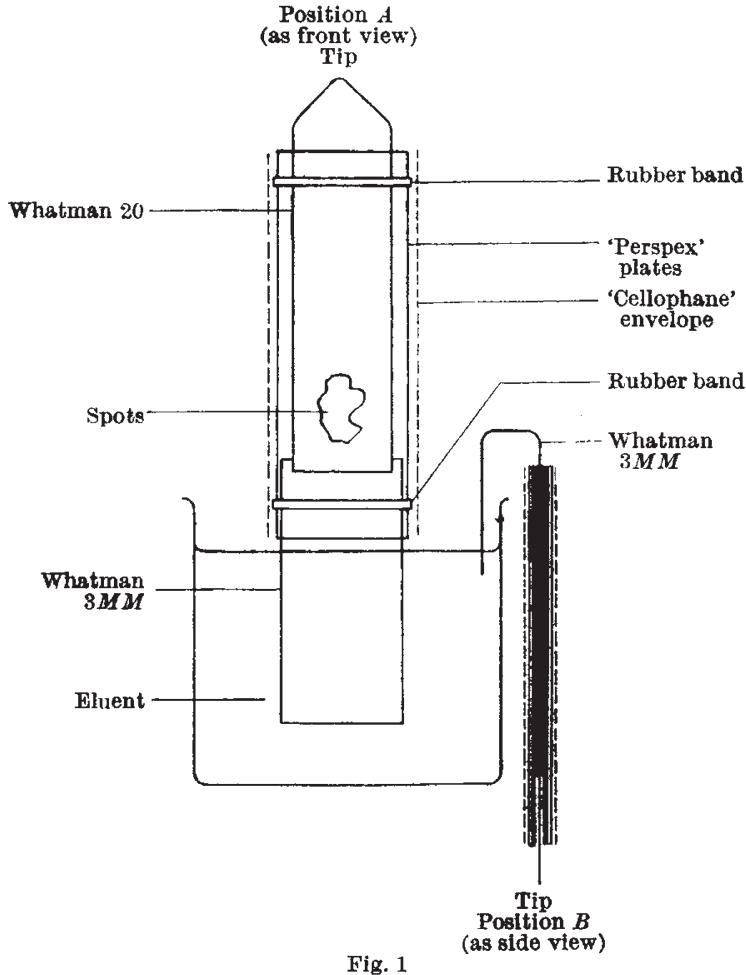

Holding the apparatus with the point upwards (position $A$ ), the ascending solvent evaporates from the tip, and passing through the spots elutes and draws the substance into the tip of the Whatman No. 20 paper. After all the substance has been concentrated in this way (usually left overnight), the apparatus is turned downwards (position $B$ ) and the substance is eluted from the tip by the descending eluant in the smallest possible amount of solvent dripping slowly from the paper. If necessary, the apparatus can stand in a box through which an inert gas can be perfused.

Experiments with dyes and other coloured substances have shown the high working efficiency of this device.

Medical Research Council

W. S. ReITH

Experimental Radiopathology Research Unit,

Hammersmith Hospital, London, W.12.

${ }^{1}$ Reith, W. S., Nature, 178, 1393 (1956).

\section{Cleavage in Chromium of High Purity}

DURING a study of the brittle fracture of chromium, we have made the following observations concerning cleavage in electro-polished test-pieces which were cut from ingots of argon arc-melted electro-deposited metal of high purity and fractured in bending at room temperature.

Cleavage occurs principally on $\{100\}$ crystallographic planes, but in many grains $\{112\}$ cleavage is also present. These two types of cleavage are readily distinguishable by microscopical examination of the fracture surface; the $\{112\}$ cleavage being in general restricted to small areas which appear as specular plane surfaces relatively free from crystallographic markings and other irregularities of the type exhibited by the $\{100\}$ cleavage facets. Less clearly defined cleavage also occurs on other crystallographic planes.

The $\{100\}$ cleavage surfaces show certain features which appear to have been observed previously only in the fracture of glass and other non-metallic materials. For example, a smooth 'mirror' surface (the smooth, relatively flat, area adjacent to the point of origin of a fracture surface in glass is always referred to as the 'mirror' of the fracture) may be present at the origin of the cleavage facet; on the 'mirror' and other smooth portions of the $\{100\}$ facets two sets of curved parallel lines, identical with the Wallner lines ${ }^{1}$ which frequently occur on fracture surfaces in glass, have also been observed. These lines originate at non-metallic inclusions or other defects in the metal which set up transverse elastic waves when the fracture front passes through them. The wave travels outwards with a constant velocity (which is a function of the physical properties of the metal) and continuously intercepts the advancing fracture front, thus momentarily deflecting the plane of cracking and producing two curved lines on the fracture surface which radiate from the originating inclusion or flaw.

The geometry of these lines shows the relationship between the velocity of fracture and the velocity of the transverse elastic wave. Smekal ${ }^{2}$ and Shand ${ }^{3}$ have given methods for determining the fracture velocity in glass from measurements made on the lines. In the case of chromium, the matter is complicated by the fact that the velocity of the transverse wave appears to vary with the crystallographic direction. It is clear, however, from the geometry of the lines that the speed of fracture increases across the 'mirror' surface to a value approximately two-thirds that of the transverse elastic wave in the direction of propagation of fracture.

It has also been observed that thin filaments may be produced during cleavage; these are generally attached at one end to the cleavage facet, and, in contrast to the bulk properties of the chromium test-piece, they show a remarkably high ductility at room temperature.

This communication is published by permission of the Chief Scientist, Department of Supply, Australia. A fuller account of the work will be published else. where.

South Australia Branch, J. F. McNerL H. R. LIMB

Defence Standards Laboratories,

C/o Post Office, Woodville,

South Australia. Dec. 24.

1 Wallner, H., Z. Phys., 144, 368 (1939).

'Smekal, A., Glastech. Ber., 23 (3), 57 (1950).

s Shand, E. B., J., Amer. Cer. Soc., 37 (12), 559 (1954).

\section{Latent Energy in Flames}

Ir has been shown ${ }^{1}$ that, to bring about ignition in an explosive medium, two conditions must be satisfied, namely, a certain temperature must be reached and a certain quantity of heat supplied. The same criteria govern change of physical state, and this presents an interesting analogy which $I$ hope to develop elsewhere.

It was further indicated that ignition is an endothermic process and that the subsequent exothermic phase of the chemical reaction belongs to the process of flame propagation rather than to that of flame 